\title{
PLASTIC MICROSTRESS FOR A DEFECT ENERGY DEPENDENT ON BURGERS TENSOR
}

\author{
Adebowale S. Borokinni \\ Mathematics Unit, Distance Learning Institute, University of Lagos, Akoka, Nigeria \\ e-mail: aborokini@unilag.edu.ng
}

\begin{abstract}
This work presents an extended form of the Aifantis strain-gradient plasticity theory through dependence of the plastic free energy on the Burgers tensor. The constraints of codirectionality for the deviatoric stress and irrotationality of the plastic distortion are assumed. These provide the basis for expressing the work done by the microstress conjugate to the Burgers tensor as the sum of the work done by the microscopic hyperstress vector and scalar. The principle of virtual power is used to establish the microforce balance, which provides the relationship between the resolved shears, plastic microstress and the microscopic hyperstresses. The microforce balance, when augmented with relevant constitutive relations that are consistent with the free-energy imbalance, results in a non-local flow rule depicted as a nonlinear second order partial differential equation in terms of the accumulated plastic strain with concomitant boundary conditions. It is shown in this work that the plastic microstress is purely dissipative and cannot account for backstress whenever the defect energy is dependent on the Burgers tensor.
\end{abstract}

Keywords: Burgers tensor, codirectionality hypothesis, strain-gradient plasticity, isotropic body, polycrystalline solid

\section{Introduction}

Strain-gradient plasticity theory deals with the study of deformation of plastically deformed bodies resulting from the development of internal microforces, which are work-conjugate to the measures of plastic strain and its gradients. In conventional or classical plasticity theories, the strain-gradient terms are not accounted for. However, the importance of strain-gradient plasticity is noted in a number of experimental results conducted over the past four decades by Ashby (1970) who showed that the flow strength of the material increases as the average particle size and spacing decrease. This observation cannot be captured by the present framework of classical plasticity. The strain-gradient plasticity theories motivated by experimental results (Ashby, 1970; Fleck et al., 1994) and the theoretical work of Aifantis (1984) and Mualhaus and Aifantis (1991), necessitate the introduction of intrinsic material length scales for dimension consistency. Conversely, the modelling of material behaviour at small length scale in the approximate range of the micron scales (i.e. say for instance, 500 nanometres to 50 micrometres) will incorporate size-dependence parameters by accounting for strain-gradient terms. In fact, it has been shown by a number of experiments that at a small length scale, the strength of metallic components during inhomogeneous plastic deformation is size-dependent with obvious phenomena that materials with smaller components are actually stronger than others (Hutchinson, 2000; Stelmashenko et al., 1993; Stolken and Evans, 1998).

The pioneer work of Aifantis (1984) on the development of strain-gradient plasticity theory which seems to generalize the classical plasticity - results in a non-local flow rule accomplanied by boundary conditions. This non-local flow rule is a nonlinear second order partial differential equation in the magnitude of the plastic strain rate. 
Equivalent form of the Aifantis flow rule was obtained by Gurtin et al. (2010) who used the codirectionality hypothesis and the Fleck-Hutchinson virtual power principle (Fleck and Hutchinson, 2001). It was assumed that the plastic microstress - which is work-conjugate to the plastic spin - expressed through its resolution to the flow direction, had energetic and dissipative parts. However, one may ask: To what extent the plastic microstress is used during plastic deformation? The answer is provided in this work.

\section{Kinematic relations}

Let $\mathbf{u}$ denote the displacement vector of an arbitrary particle in a plastically deformed polycrystalline solid body $B$ undergoing infinitesimal deformation, then the gradient of displacement vector $\nabla \mathbf{u}$ has the definition ${ }^{1}$

$$
\nabla \mathbf{u}=\mathbf{H}^{e}+\mathbf{H}^{p} \quad \operatorname{tr} \mathbf{H}^{p}=0
$$

where $\mathbf{H}^{e}$ is the elastic part of the displacement gradient which characterizes stretching and rotation of the material structure, while $\mathbf{H}^{p}$ is called the plastic distortion and characterizes the defect through the material structure. Taking the symmetric part of $(2.1)$ we have ${ }^{2}$

$$
\operatorname{sym} \nabla \mathbf{u}=\mathbf{E}^{e}+\mathbf{E}^{p} \quad \operatorname{tr} \mathbf{E}^{p}=0
$$

where $\mathbf{E}^{e}=\operatorname{sym} \mathbf{H}^{e}$ is the elastic strain and $\mathbf{E}^{p}=\operatorname{sym} \mathbf{H}^{p}$ is called the plastic strain.

The accumulated plastic strain $e^{p}$ is defined via the plastic strain rate as

$$
\dot{e}^{p}=\left|\dot{\mathbf{E}}^{p}\right|
$$

with the given initial condition

$$
e^{p}(\mathbf{X}, 0)=0
$$

By (2.3) and (2.4) it is clear that the time derivative $\dot{e}^{p}(\mathbf{X}, t) \geqslant 0$ for all time $t$. Thus, $e^{p}(\mathbf{X}, t)$ is an increasing function of time.

Let $\mathbf{N}^{p}$ denote the flow direction defined by

$$
\mathbf{N}^{p}=\frac{\dot{\mathbf{E}}^{p}}{\left|\dot{\mathbf{E}}^{p}\right|} \quad \text { for } \quad \dot{\mathbf{E}}^{p} \neq \mathbf{0}
$$

Whenever $\left|\dot{\mathbf{E}}^{p}\right| \neq 0$, then there is flow. $\dot{\mathbf{E}}^{p}$ in terms of the accumulated plastic strain rate $\dot{e}^{p}$ and the flow direction is given by

$$
\dot{\mathbf{E}}^{p}=\dot{e}^{p} \mathbf{N}^{p}
$$

Given that $\mathbf{T}$ is the elastic macrostress (which is symmetric) then the codirectionality hypothesis asserts that the direction of the plastic strain rate coincides with the direction of the deviatoric stress such that

$$
\frac{\mathbf{T}_{o}}{\left|\mathbf{T}_{o}\right|}=\frac{\dot{\mathbf{E}}^{p}}{\left|\dot{\mathbf{E}}^{p}\right|}=\mathbf{N}^{p}
$$

This is called the codirectionality constraint.

On the basis of (2.5), sym $\nabla \dot{\mathbf{u}}$ can be written as

$$
\operatorname{sym} \nabla \dot{\mathbf{u}}=\dot{\mathbf{E}}^{e}+\dot{e}^{p} \mathbf{N}^{p} \quad \text { with } \quad \operatorname{tr} \mathbf{N}^{p}=0
$$

\footnotetext{
${ }^{1}$ The gradient of a vector $\mathbf{a}$ would be denoted as $\nabla \mathbf{a}$ and would be written in component form as $a_{i, j}=\partial a_{i} / \partial x_{j}$ for $i, j=1,2,3$. The gradient of a scalar field $\phi$ would be denoted as $\nabla \phi$ and written as $\phi, i=\partial \phi / \partial x_{i}$.

${ }^{2}$ The symmetric part of the second order tensor $\mathbf{A}$ is defined by $\operatorname{sym} \mathbf{A}=\left(\mathbf{A}+\mathbf{A}^{\mathrm{T}}\right) / 2$, where $\mathbf{A}^{\mathrm{T}}$ is the transpose of the tensor $\mathbf{A}$. In component form, we have $(\operatorname{sym} \mathbf{A})_{i j}=\left(A_{i j}+A_{j i}\right) / 2$.
} 


\section{Streamline virtual power principle and force balances}

The streamline virtual principle is based on the power balance principle in which the stress $\mathbf{T}$ is known to be symmetric a priori. Also the plastic strain rate is written in terms of the accumulated plastic strain rate. A theory not accounting for the plastic strain gradient has the streamline power balance to be written as $^{3}$

$$
\int_{P} \mathbf{b} \cdot \dot{\mathbf{u}} d V+\int_{\partial P} \mathbf{t} \cdot \dot{\mathbf{u}} d A=\int_{P} \mathbf{T}: \dot{\mathbf{E}}^{e} d V+\int_{P} \tau^{p} \dot{e}^{p} d V
$$

where $\mathbf{b}$ is the body force on a subregion $P$ of $B$, and $\mathbf{t}$ is the macrotraction force on the boundary $\partial P$, so that the sum of integrals on the left handside of (3.1) is called the power-expenditure by external forces. Also $\tau^{p}$ is the plastic stress resolved to the flow direction. $\tau^{p}$ is assumed to be power-conjugate to the accumulated plastic strain rate. The sum of integral at the right hand side of (3.1) is called the power expenditure by the internal microstresses.

Our objective is to formulate a model that accounts for the plastic strain gradient. We will do this by introducing for the Burgers tensor without plastic rotation. This assumption is motivated from the knowledge of the fact that dislocation in the material arising from plasticity theory is also explsined through the Burgers vector. However, a plasticity theory based on the Burgers tensor must necessarily take into account the plastic rotation, except if from the outset one assumes that there is not plastic rotation. Most strain gradient theories however, do not accept the plastic rotation and, also because for reason of complexity in the flow rule, we will assume the absence of plastic rotation.

The Burgers tensor is defined by (in the absence of plastic rotation) ${ }^{4}$

$$
\mathbf{G}=\operatorname{Curl} \mathbf{E}^{p}
$$

The Burgers tensor rate in terms of the accumulated plastic strain rate $\dot{e}^{p}$ and the flow direction $\mathbf{N}^{p}$ following (2.6) is

$$
\dot{\mathbf{G}}=\operatorname{Curl}\left(\dot{e}^{p} \mathbf{N}^{p}\right)
$$

In component form and using the product rule of differentiation, we have

$$
\dot{G}_{i j}=\epsilon_{i p q} \dot{e}_{{ }_{p}}^{p} N_{j q}^{p}+\epsilon_{i p q} \dot{e}^{p} N_{j q, p}^{p}
$$

In invariant form we have ${ }^{5}$

$$
\dot{\mathbf{G}}=\left(\nabla \dot{e}^{p} \times\right) \mathbf{N}^{p}+\dot{e}^{p} \operatorname{Curl} \mathbf{N}^{p}
$$

Since $\mathbf{G}$ is a geometric quantity, there exists a microstress $\mathbf{S}$ power-conjugate to $\dot{\mathbf{G}}$. Thus, we can write $\mathbf{S}: \dot{\mathbf{G}}$ as

$$
\mathbf{S}: \dot{\mathbf{G}}=\mathbf{S}:\left(\nabla \dot{e}^{p} \times\right) \mathbf{N}^{p}+\left(\mathbf{S}: \operatorname{Curl} \mathbf{N}^{p}\right) \dot{e}^{p}
$$

This implies that we have

$$
\mathbf{S}: \dot{\mathbf{G}}=S_{i j} \epsilon_{i p q} N_{j q} \dot{e}_{, p}^{p}+S_{i j} \epsilon_{i p q} N_{j q, p}^{p} \dot{e}^{p}
$$

\footnotetext{
${ }^{3}$ The inner products $\mathbf{a} \cdot \mathbf{b}$ and $\mathbf{A}: \mathbf{B}$ are defined as $a_{i} b_{i}$ and $A_{i j} B_{i j}$, respectively.

${ }^{4}$ The component of the curl of the tensor field $\mathbf{A}$ denoted as $\operatorname{Curl} \mathbf{A}$ or $\nabla \times \mathbf{A}$ is expressed as $\epsilon_{i p q} A_{j q, p}$ where, $\epsilon_{i p q}$ is the permutation symbol.

${ }^{5}$ Given a nonzero vector a, the component of the second order tensor $(\mathbf{a} \times)$ is expressed as $\epsilon_{i k j} a_{k}$.
} 
Let the vector $\boldsymbol{\xi}$ and the scalar $\phi$ be defined as

$$
\xi_{k}=S_{i j} \epsilon_{i k q} N_{j q}^{p} \quad \phi=S_{i j} \epsilon_{i p q} N_{j q, p}^{p}
$$

then by definition of $\boldsymbol{\xi}$ and $\phi$ in (3.5), we have

$$
\mathbf{S}: \dot{\mathbf{G}}=\boldsymbol{\xi} \cdot \nabla \dot{e}^{p}+\phi \dot{e}^{p}
$$

We shall refer to $\boldsymbol{\xi}$ and $\phi$ as the microscopic hyperstress vector and microscopic stress scalar, respectively. Following Gurtin (2004), the power balance in the absence of plastic spin in the Burgers tensor rate $\dot{\mathbf{G}}$ is given $b^{6}$

$$
\int_{P} \mathbf{b} \cdot \dot{\mathbf{u}} d V+\int_{\partial P} \mathbf{t}(\mathbf{n}) \cdot \dot{\mathbf{u}} d A=\int_{P}\left[\mathbf{T}: \dot{\mathbf{E}}^{e}+\tau^{p} \dot{e}^{p}+\mathbf{S}: \dot{\mathbf{G}}\right] d V
$$

By (3.6), we have

$$
\int_{P} \mathbf{b} \cdot \dot{\mathbf{u}} d V+\int_{\partial P} \mathbf{t}(\mathbf{n}) \cdot \dot{\mathbf{u}} d A=\int_{P}\left[\mathbf{T}: \dot{\mathbf{E}}^{e}+\tau^{p} \dot{e}^{p}+\boldsymbol{\xi} \cdot \nabla \dot{e}^{p}+\phi \dot{e}^{p}\right] d V
$$

Since the term $\mathbf{T}: \operatorname{sym} \nabla \dot{\mathbf{u}}$, when $\dot{\mathbf{E}}^{p}=\mathbf{0}$ would give a rise to the boundary term $\mathbf{T n} \cdot \dot{\mathbf{u}}$ leading to the macrotraction condition $\mathbf{T n}=\mathbf{t}(\mathbf{n})$, then the term $\boldsymbol{\xi} \cdot \nabla \dot{e}^{p}$ would give a rise to the traction condition, so that we can slightly modify power balance (3.8) to take into account the microtraction scalar $\eta(\mathbf{n})$ power-conjugate to $\dot{e}^{p}$ on $\partial P$. Thus, (3.8) will take the form

$$
\int_{P} \mathbf{b} \cdot \dot{\mathbf{u}} d V+\int_{\partial P} \mathbf{t}(\mathbf{n}) \cdot \dot{\mathbf{u}} d A+\int_{\partial P} \eta(\mathbf{n}) \dot{e}^{p} d A=\int_{P}\left[\mathbf{T}: \dot{\mathbf{E}}^{e}+\tau^{p} \dot{e}^{p}+\phi \dot{e}^{p}+\boldsymbol{\xi} \cdot \nabla \dot{e}^{p}\right] d V
$$

By using the virtual velocity $\nu$ in the list of basic rate-like kinematic variables $\nu=\left(\widetilde{\mathbf{u}}, \widetilde{\mathbf{E}}^{e}, \widetilde{e}^{p}\right)$ satisfying

$$
\operatorname{sym} \nabla \widetilde{\mathbf{u}}=\mathbf{E}^{e}+\widetilde{e} \mathbf{N}
$$

we have the virtual power which takes the form

$$
\int_{P} \mathbf{b} \cdot \widetilde{\mathbf{u}} d V+\int_{\partial P} \mathbf{t}(\mathbf{n}) \cdot \widetilde{\mathbf{u}} d A+\int_{\partial P} \eta(\mathbf{n}) \widetilde{e}^{p} d A=\int_{P}\left[\mathbf{T}: \widetilde{\mathbf{E}}^{e}+\tau^{p} \widetilde{e}^{p}+\boldsymbol{\xi} \cdot \nabla \widetilde{e}^{p}+\phi \widetilde{e}^{p}\right] d V
$$

By assuming that $\widetilde{e}^{p}=0$ consistent with (3.10) we have

$$
\int_{P} \mathbf{b} \cdot \widetilde{\mathbf{u}} d V+\int_{\partial P} \mathbf{t}(\mathbf{n}) \cdot \widetilde{\mathbf{u}} d A=\int_{P} \mathbf{T}: \nabla \widetilde{\mathbf{u}} d V
$$

Applying the divergence theorem, we have ${ }^{7}$

$$
\int_{P}(\operatorname{Div} \mathbf{T}+\mathbf{b}) \cdot \widetilde{\mathbf{u}} d V=\int_{\partial P}(\mathbf{T n}-\mathbf{t}(\mathbf{n})) \cdot \widetilde{\mathbf{u}} d A
$$

\footnotetext{
${ }^{6}$ In Gurtin $(2004) \tau^{p} \dot{e}^{p}$ is written as $\mathbf{T}: \dot{\mathbf{E}}^{p}$. In this work, we note that $\tau^{p}=\mathbf{T}^{p}: \mathbf{N}^{p}$ so that $\tau^{p} \dot{e}^{p}=\mathbf{T}^{p}: \dot{e}^{p} \mathbf{N}^{p}=\mathbf{T}^{p}: \dot{\mathbf{E}}^{p}$, where $\mathbf{T}^{p}$ is called the plastic microstress.

${ }^{7}$ The divergence of the second order tensor field $\mathbf{A}$ denoted as Div $\mathbf{A}$ is expressed in component form as $A_{i j, j}$, while the divergence of the vector a denoted as Div $\mathbf{a}$ is expressed as $a_{k, k}$.
} 
By fundamental lemma on calculus of variation, we have the following macroscopic force balance

$$
\operatorname{Div} \mathbf{T}+\mathbf{b}=\mathbf{0} \quad \text { in } P
$$

and the macrotraction condition given by

$$
\mathbf{T n}=\mathbf{t}(\mathbf{n}) \quad \text { on } \partial P
$$

Consistently with (3.10), assume that $\widetilde{\mathbf{u}}=\mathbf{0}$, so that the virtual power balance becomes

$$
\int_{\partial P} \eta(\mathbf{n}) \widetilde{e}^{p} d A=\int_{P}\left[\mathbf{T}: \widetilde{\mathbf{E}}^{e}+\tau^{p} \widetilde{e}^{p}+\boldsymbol{\xi} \cdot \nabla \widetilde{e}^{p}+\phi \widetilde{e}^{p}\right] d V
$$

Since $\widetilde{\mathbf{u}}=\mathbf{0}$ then $\widetilde{\mathbf{E}}^{e}=-\widetilde{e}^{p} \mathbf{N}^{p}$. Thus we can write

$$
\mathbf{T}: \widetilde{\mathbf{E}}^{e}=-\left(\mathbf{T}: \mathbf{N}^{p}\right) \widetilde{e}^{p}=-\tau \widetilde{e}^{p}
$$

where $\tau=\mathbf{T}: \mathbf{N}^{p}$ is called the resolved shears. (3.16) can be written as

$$
\int_{\partial P} \eta(\mathbf{n}) \widetilde{e}^{p} d A=\int_{P}\left[\left(\tau^{p}+\phi-\tau\right) \widetilde{e}^{p}+\boldsymbol{\xi} \cdot \nabla \widetilde{e}^{p}\right] d V
$$

By the divergence theorem, (3.17) becomes

$$
\int_{\partial P}(\eta(\mathbf{n})-\boldsymbol{\xi} \cdot \mathbf{n}) \widetilde{e}^{p} d A=\int_{P}\left[\tau^{p}+\phi-\tau-\operatorname{Div} \boldsymbol{\xi}\right] \widetilde{e}^{p} d V
$$

By fundamental lemma on calculus of variation, we have the microscopic force balance given as

$$
\tau=\tau^{p}+\phi-\operatorname{Div} \boldsymbol{\xi}
$$

with the microtraction condition given as

$$
\boldsymbol{\xi} \cdot \mathbf{n}=\eta(\mathbf{n})
$$

\section{Free-energy imbalance}

Here we assume that the free-energy function $\psi=\widehat{\psi}\left(\mathbf{E}^{e}, \mathbf{G}\right)$ is a function of the elastic strain and the Burgers tensor. But since the inner product $\mathbf{S}: \dot{\mathbf{G}}$ can be written in terms of the accumulated plastic strain rate and its gradient with the knowledge that the energetic part of $\mathbf{S}$ is the derivative of the free-energy with respect to the Burgers tensor (cf. Gurtin, 2004; Poh and Peerling, 2016). We can assume the free energy as a function of the elastic strain, accumulated plastic strain and the gradient of the accumulated plastic strain, i.e.

$$
\psi=\widehat{\psi}\left(\mathbf{E}^{e}, e^{p}, \nabla e^{p}\right)
$$

The free-energy imbalance states that the rate of increase of the total free-energy in the body cannot exceed the total power expenditure by external forces. A consequence of this is the inequality which notes the power balance principle

$$
\int_{P} \dot{\psi} d V \leqslant \int_{P}\left[\mathbf{T}: \dot{\mathbf{E}}^{e}+\tau^{p} \dot{e}^{p}+\phi^{p} \dot{e}^{p}+\boldsymbol{\xi} \cdot \nabla \dot{e}^{p}\right] d V
$$

In local form (4.2) reduces to the inequality

$$
\dot{\psi}-\mathbf{T}: \dot{\mathbf{E}}^{e}-\tau^{p} \dot{e}^{p}-\boldsymbol{\xi} \cdot \nabla \dot{e}^{p}=-\delta \leqslant 0
$$

where $\delta$ is called the dissipation. This inequality would be used to derive the constitutive relations for the microstresses. 


\section{Constitutive relations}

We shall assume that the free-energy admits the decomposition

$$
\psi=\widehat{\psi}\left(\mathbf{E}^{e}, e^{p}, \nabla e^{p}\right)=\widehat{\psi}^{e}\left(\mathbf{E}^{e}\right)+\widehat{\psi}^{p}\left(e^{p}, \nabla e^{p}\right)
$$

into elastic and plastic parts, respectively. Since the Burgers tensor $\mathbf{G}$ relates the accumlated plastic strain $e^{p}$ and its gradient $\nabla e^{p}$ from (3.4), we can write the plastic free energy $\widehat{\psi}^{p}\left(e^{p}, \nabla e^{p}\right)$ as a function of the Burger tensor through the assumed relation

$$
\widehat{\psi}^{p}\left(e^{p}, \nabla e^{p}\right)=\widehat{\psi}_{G}^{p}(\mathbf{G})
$$

Consistent with the free-energy imbalance (4.3), we have

$$
\left(\frac{\partial \psi^{e}}{\partial \mathbf{E}^{e}}-\mathbf{T}\right): \dot{\mathbf{E}}^{e}+\left(\frac{\partial \widehat{\psi}^{p}}{\partial e^{p}}-\tau^{p}-\phi\right) \dot{e}^{p}+\left(\frac{\partial \widehat{\psi}^{p}}{\partial e^{p}}-\boldsymbol{\xi}\right) \cdot \nabla \dot{e}^{p} \leqslant 0
$$

Using the Coleman-Noll procedure, it is conventional to have the elastic stress as

$$
\mathbf{T}=\frac{\partial \widehat{\psi}^{e}}{\partial \mathbf{E}^{e}}
$$

In this present work, we assume that the microstress $\mathbf{S}$ contains the energetic part $\mathbf{S}^{e n}$ and the dissipative part $\mathbf{S}^{d i s}$. It has been established that the energetic microstress $\mathbf{S}^{e n}$ is (see Gurtin, 2004)

$$
\mathbf{S}^{e n}=\frac{\partial \widehat{\psi}_{G}^{p}(\mathbf{G})}{\partial \mathbf{G}}
$$

Given that $\phi^{e n}$ and $\boldsymbol{\xi}^{e n}$ are the energetic parts of $\phi$ and $\boldsymbol{\xi}$, then it is obvious that

$$
\phi^{e n}=\frac{\partial \widehat{\psi}^{p}}{\partial e^{p}} \quad \boldsymbol{\xi}=\frac{\partial \widehat{\psi}^{p}}{\partial \nabla e^{p}}
$$

Equation (5.6) is obtained from the fact that

$$
\dot{\psi}^{p}=\dot{\psi}_{G}^{p}=\mathbf{S}^{e n}: \dot{\mathbf{G}}=\phi^{e n} \dot{e}^{p}+\boldsymbol{\xi}^{e n} \cdot \nabla \dot{e}^{p}=\frac{\partial \widehat{\psi}^{p}}{\partial e^{p}} \dot{e}^{p}+\frac{\partial \widehat{\psi}^{p}}{\partial \nabla e^{p}} \cdot \nabla \dot{e}^{p}
$$

It would be assumed that the microstresses $\phi$ and $\boldsymbol{\xi}$ can be additively decomposed as

$$
\phi=\phi^{e n}+\phi^{d i s} \quad \boldsymbol{\xi}=\boldsymbol{\xi}^{e n}+\boldsymbol{\xi}^{d i s}
$$

where $\phi^{d i s}$ is the dissipative part of $\phi$ and $\boldsymbol{\xi}^{\text {dis }}$ is the dissipative part of $\boldsymbol{\xi}$.

By substituting (5.4) and (5.6) into inequality (5.3), we have the dissipation inequality given as

$$
\delta=\tau^{p} \dot{e}^{p}+\phi^{d i s} \dot{e}^{p}+\boldsymbol{\xi}^{d i s} \cdot \nabla \dot{e}^{p} \geqslant 0
$$

Remark: From this inequality, it is obvious that the microstress $\tau^{p}$ is purely dissipative and cannot explain the backstress whenever the defect energy is dependent on the Burgers tensor (see Gurtin et al., 2010; Han and Reddy, 2013). This also implies that the unresolved plastic stress $\mathbf{T}^{p}$ (see footnote 6 ) is purely dissipative. 


\section{Flow rule}

Microforce balance (3.19) can be written as

$$
\tau-\left(\phi^{e n}-\operatorname{Div} \boldsymbol{\xi}^{e n}\right)=\tau^{p}+\phi^{d i s}-\operatorname{Div} \boldsymbol{\xi}^{d i s}
$$

The term in bracket of the left hand side of (6.1) is called the backstress. The general flow rule using equations (5.6) and (5.7) is

$$
\tau-\left[\frac{\partial \widehat{\psi}^{p}}{\partial e^{p}}-\operatorname{Div}\left(\frac{\partial \widehat{\psi}^{p}}{\partial \nabla e^{p}}\right)\right]=\widehat{\tau}^{p}\left(e^{p}, \nabla e^{p}\right)+\phi^{d i s}\left(e^{p}, \nabla e^{p}\right)-\operatorname{Div} \boldsymbol{\xi}^{d i s}
$$

Based on the Aifantis model, it would be assumed that $\widehat{\tau}^{p}\left(e^{p}, \nabla e^{p}\right)=Y\left(e^{p}\right)$ where $Y\left(e^{p}\right)>0$ is called the coarse-grain flow resistance. In Gurtin et al. (2010), it is assumed that the plastic free energy can take the form

$$
\widehat{\psi}^{p}\left(e^{p}, \nabla e^{p}\right)=\frac{1}{2} \beta\left(e^{p}\right)\left|\nabla e^{p}\right|^{2}
$$

with the condition that $\beta\left(e^{p}\right)>0$ and $\frac{d \beta\left(e^{p}\right)}{d e^{p}} \geqslant 0$; so that if we let $\mathbf{g}=\nabla e^{p}$ then

$$
\phi^{e n}=\frac{1}{2} \frac{d \beta\left(e^{p}\right)}{d e^{p}}|\mathbf{g}|^{2} \quad \boldsymbol{\xi}^{e n}=\beta\left(e^{p}\right) \mathbf{g}
$$

Borokinni and Ajayi (2017) showed that $\boldsymbol{\xi}^{\text {dis }}=\mathbf{0}$ and $\phi^{\text {dis }}=0$ (so that $\mathbf{S}$ is purely energetic) given codirectionality constraint (2.7). Thus, a more general form of the Aifantis flow rule is given $\operatorname{as}^{8}$

$$
\tau-\left(\frac{1}{2} \beta^{\prime}\left(e^{p}|\mathbf{g}|^{2}-\operatorname{Div}\left(\beta\left(e^{p}\right) \mathbf{g}\right)\right)\right)=Y\left(e^{p}\right)
$$

where $\beta^{\prime}\left(e^{p}\right)=d \beta\left(e^{p}\right) / d e^{p}$. By the rule of differentiation, it is obvious that

$$
\operatorname{Div}\left(\beta\left(e^{p}\right) \mathbf{g}\right)=\beta\left(e^{p}\right) \operatorname{Div} \mathbf{g}+\beta^{\prime}\left(e^{p}\right)|\mathbf{g}|^{2}=\beta\left(e^{p}\right) \Delta e^{p}+\beta^{\prime}\left(e^{p}\right)\left|\nabla e^{p}\right|^{2}
$$

By substituting (6.6) into (6.5), we get a modified Aifantis flow rule given as

$$
\tau-\left(\frac{1}{2} \beta^{\prime}\left(e^{p}\right)\left|\nabla e^{p}\right|^{2}+\beta\left(e^{p}\right) \Delta e^{p}\right)=Y\left(e^{p}\right)
$$

The term $\beta^{\prime}\left(e^{p}\right)\left|\nabla e^{p}\right|^{2} / 2$ describes softening behaviour.

\section{Simple boundary condition and variational formulation of the flow rule}

Consider microtraction condition (3.20). The global power expenditure by the microtraction $\eta(\mathbf{n})$ on the boundary $\partial B$ of the body $B$ is given as

$$
\int_{\partial B}(\boldsymbol{\xi} \cdot \mathbf{n}) \dot{e}^{p} d A=\int_{\partial B} \eta(\mathbf{n}) \dot{e}^{p} d A
$$

For a simple boundary condition, we assume that the power expenditure by the microtraction $\eta(\mathbf{n})$ is zero, so that in local form we have

$$
(\boldsymbol{\xi} \cdot \mathbf{n}) \dot{e}^{p}=0 \quad \text { on } \quad \partial B
$$

\footnotetext{
${ }^{8}$ In the Aifantis model, $\beta$ is assumed to be a constant.
} 
For microscopically simple boundary conditions, we have

$$
\dot{e}^{p}=0 \quad \text { on } \quad \Gamma_{\text {hard }} \quad \boldsymbol{\xi} \cdot \mathbf{n}=0 \quad \text { on } \quad \Gamma_{\text {free }}
$$

where $\Gamma_{\text {hard }}$ and $\Gamma_{\text {free }}$ are complementary subsurfaces called the microscopically hard and free surfaces, respectively. $\Gamma_{\text {hard }}$ is that part of the boundary $\partial B$ which does not allow the flow of dislocation while $\Gamma_{\text {free }}$ is the part of $\partial B$ that allows the flow of dislocation through the boundary $\partial B$.

Next, we show that given a test field $f$ satisfying $f=0$ on $\Gamma_{\text {hard }}$, then the weak form

$$
\int_{B}\left[\tau^{p} f-\tau f+\phi f+\boldsymbol{\xi} \cdot \nabla f\right] d V=0
$$

is equivalent to microforce balance (3.19) with the microtraction condition

$$
\boldsymbol{\xi} \cdot \mathbf{n}=0 \quad \text { on } \quad \Gamma_{\text {free }}
$$

The use of the divergence theorem implies that (7.4) becomes

$$
\int_{B}\left[\tau^{p}-\tau+\phi-\operatorname{Div} \boldsymbol{\xi}\right] f d V+\int_{\Gamma_{\text {free }}}(\boldsymbol{\xi} \cdot \mathbf{n}) f d A=0
$$

By fundamental lemma on calculus of variation, it is obvious that (3.19) and (7.5) are satisfied. Hence, assuming that the constitutive equations for the microstresses and the macrostress hold, then flow rule (6.2) on $B$ and boundary conditions (7.3) are together equivalent to the requirement that (7.4) is satisfied for all test fields.

\section{Plastic free-energy balance}

Assume the null expenditure of microscopic power with simple microscopic boundary condition (7.3), then

$$
\begin{aligned}
\int_{B} \dot{\vec{\psi}}^{p}\left(e^{p}, \nabla e^{p}\right) d V=\int_{B}\left(\phi^{e n} \dot{e}^{p}+\boldsymbol{\xi}^{e n} \cdot \nabla \dot{e}^{p}\right) d V \\
\quad=\int_{B}\left(\phi \dot{e}^{p}+\boldsymbol{\xi}^{p} \cdot \nabla \dot{e}^{p}\right) d V-\int_{B}\left(\phi^{d i s} \dot{e}^{p}+\boldsymbol{\xi}^{d i s} \cdot \nabla \dot{e}^{p}\right) d V
\end{aligned}
$$

By the divergence theorem, and noting the assumption of the null microscopic powerexpenditure, we have

$$
\int_{B} \dot{\vec{\psi}}^{p}\left(e^{p}, \nabla e^{p}\right) d V=\int_{B}(\phi-\operatorname{Div} \boldsymbol{\xi}) \dot{e}^{p} d V-\int_{B}\left(\phi^{d i s} \dot{e}^{p}+\boldsymbol{\xi}^{d i s} \cdot \nabla \dot{e}^{p}\right) d V
$$

Recall that the microforce balance has form (3.19), so that we have

$$
\int_{B} \dot{\vec{\psi}}^{p}\left(e^{p}, \nabla e^{p}\right) d V=\int_{B} \tau \dot{e}^{p} d V-\int_{B}\left(\tau^{p} \dot{e}^{p}+\phi^{d i s} \dot{e}^{p}+\boldsymbol{\xi}^{d i s} \cdot \nabla \dot{e}^{p}\right) d V
$$

Equation (8.3) is the plastic free-energy balance, and the consequence of this balance is that the rate of increase in the defect energy $\widehat{\psi}^{p}$ is less or equal to the plastic working ${ }^{9}$. Thus, we have the inequality

$$
\int_{B} \dot{\widehat{\psi}}\left(e^{p}, \nabla \dot{e}^{p}\right) d V \leqslant \int_{B} \tau \dot{e}^{p} d V
$$

The integral on the right-hand side of inequality (8.4) is called the plastic working.

\footnotetext{
${ }^{9}$ It should be recalled by (5.8) that the dissipation is nonnegative
} 


\section{Conclusion}

This work has shown that the work done by the microstress $\mathbf{S}$ - work-conjugate to the Burgers tensor $\mathbf{G}=\nabla \times \mathbf{E}^{p}$ - admits the additive decomposition into the work done by a microstress scalar $\phi$ and a microscopic hyperstress $\boldsymbol{\xi}$ which are work-conjugate to the accumulated plastic strain and its gradient, respectively. This formulation leads to microscopic force balance for the associated system of microforces. The microforce balance is augmented with the constitutive relations for the microforces to obtain the flow rule, in the form of a partial differential equation generalizing the Aifantis flow rule. It is shown that the plastic microstress is dissipative while the energetic contribution to the flow rule comes from the microstress $\mathbf{S}$ via $\phi$ and $\boldsymbol{\xi}$.

\section{References}

1. Aifantis E.C., 1984, On the microstructural origin of certain inelastic models, Journal of Engineering Materials and Technology, Transactions of the ASME, 106, 326-330

2. Ashby M.F., 1970, The deformation of plastically non-homogeneous materials, Philosophical Magazine, 21, 399-424

3. Borokinni A.S., AJAYI K.F., 2017, On Aifantis' strain gradient plasticity theory accounting for plastic spin, Mechanics Research Communications, 84, 110-115

4. Fleck N.A., Hutchinson J.W., 2001, A reformulation of strain gradient plasticity, Journal of the Mechanics and Physics of Solids, 49, 2245-2271

5. Fleck N.A., Muller G.M., Ashby M.F., Hutchinson J.W., 1994, Strain gradient plasticity: theory and experiment, Acta Metallurgica et Materialia, 42, 2, 475-487

6. GuRTin M.E., 2004, A gradient theory of small-deformation isotropic plasticity that accounts for Burgers vector and dissipation due to plastic spin, Journal of the Mechanics and Physics of Solids, 52, 2545-2568

7. Gurtin M.E., Fried E., Anand L., 2010, Mechanics and Thermodynamics of Continua, Cambridge University Press, Cambridge

8. Han W., Reddy B.D., 2013, Plasticity: Mathematical Theory and Numerical Analysis, New York: Springer-Velag

9. Hutchinson J.W., 2000, Plasticity at the micron scale, International Journal of Solids and Structures, $\mathbf{3 7}, 225-238$

10. Mualhaus H.B., Aifantis E.C., 1991, A variational principle for gradient plasticity, International Journal of Solids and Structures, 28, 845-857

11. Рoh L.H., Peerlings R.H.J., 2016, The plastic rotation effect in an isotropic gradient plasticity model for applications at the meso scale, International Journal of Solids and Structures, 78-79, 75-69

12. Stelmashenko N.A., Wallis M.G., Brown L.M., Milman Y.V., 1993. Microidentations on W and Mo oriented single crystals: An STM study, Acta Metallurgica et Materialia, 41, 2855-2865

13. Stolken J.S., Evans A.G., 1998, A microbend test method for measuring the plasticity length scale, Acta Materialia, 46, 5109-5115 